\title{
Investigation on the Knowledge of Abnormal Characteristics of Long Compartment Cylindrical Shell with Ring-Stiffeners in the Course of Submarine Strength
}

Weijun $\mathrm{Xu}^{1, \mathrm{a},{ }^{*}}$, Huilong Ren ${ }^{1}$, Chenfeng $\mathrm{Li}^{1}$, Guoqing Feng ${ }^{1}$, Xueqian Zhou ${ }^{1}$ and Ning $\mathrm{Liu}^{1}$

${ }^{I}$ College of Shipbuilding Engineering, Harbin Engineering University, Harbin, Heilongjiang, P.R.China

axuweijun@hrbeu.edu.cn

*Corresponding author

\begin{abstract}
The stability of submarine pressure cylindrical shell is the main factor that restricts the diving of submarine for a long time, and it is also one of the important contents for designing and checking of submarine pressure structure. It is well known that a way to improve the stability of submarine pressure cylindrical shell is increasing the thickness or improve the ring stiffness, in fact, the increasing thickness is a kind of effective method to improve the stability of ring-stiffened cylindrical shell with long compartment, however, the improvement of overall stability for the cylindrical shell cannot achieve by increasing its ring stiffness, which can be regarded as the "Abnormal characteristics" appears in the structure. This paper mainly investigates the abnormal characteristics of the ring-stiffened cylindrical shell with a long compartment, which should be mastered by undergraduate students majoring in Naval Architecture and Ocean Engineering. The investigation results provide references for the reform of the course system of structural mechanics of underwater pressure shell.
\end{abstract}

Keywords: ring stiffened cylindrical shell with long compartment, abnormal characteristics, submarine strength, reform of course system

\section{《潜艇强度》课程中关于长舱段环肋圆柱壳异常特性的 知识探究}

\author{
许维军 ${ }^{1, \mathrm{a}^{*}}$, 任慧龙 ${ }^{1}$, 李陈峰 ${ }^{1}$, 冯国庆 ${ }^{1}$, 周学谦 ${ }^{1}$, 刘宁 ${ }^{1}$
}

I 船舶工程学院, 哈尔滨工程大学, 哈尔滨, 黑龙江, 中国

axuweijun@hrbeu.edu.cn

*通讯作者

摘要

潜艇耐压圆柱壳结构的稳定性问题是长期以来制约潜艇下潜的主要因素，也是潜艇耐压结构设计与校 核的重要内容之一。众所周知，提高潜艇耐压圆柱壳结构的稳定性途径是通过增加板厚或者提高环向 刚度, 对于长舱段环肋圆柱壳增加板厚是一种有效的改善稳定性的方法, 但是增加环向刚度却不能有 效地改善结构的总体稳定性, 这种情况下可以认为结构出现了 “异常特性”。本文通过对船舶与海洋 工程专业本科生所应掌握的潜艇耐压长舱段环肋圆柱壳异常特性的知识进行探索, 为水下耐压壳体结 构力学方向课程体系改革提供参考。

关键词: 长舱段环肋圆柱壳, 异常特性, 潜艇强度, 课程体系改革

\section{1. 引言}

潜艇服役过程中, 耐压环肋圆柱壳结构的失稳是潜艇耐压
结构破坏的主要模式之一, 其包含了壳板失稳, 肋骨失稳 和总体失稳三种失稳情况 [1]。为了保证潜艇发挥应有的 巡航和作战性能, 稳定性校核已经成为必要的校核内容, 尤其是潜深比较大的情况下, 结构的强度问题反而不如稳 
解现代大型潜艇的发展方向。但当前本科生《潜艇强度》 一书所述的潜艇相关内容主要为潜艇结构设计中的基础 知识, 虽然为《潜艇强度》课程教学打下了坚实的基础, 但其内容多年尚未更新, 在某种程度已经不能反映现代大 型潜艇设计过程中需要面对的问题。尤其是长舱段环肋圆 柱壳的使用, 其力学性能在某种程度应用《潜艇强度》一 书中所述的内容已不能很好地解释。因此, 有必要引入新 的相关内容丰富本科生的相关课程教学。

《潜艇强度》课程主要讲解潜艇结构设计的有关方法, 内 容主要包括潜艇耐压结构强度与稳定性的计算与校核。通 过本课程的学习, 使学生能掌握潜艇结构设计的基本方 法, 使其初步具有设计潜艇耐压结构的能力。本课程共计 24 课时, 在课程内容的安排上应遵循如下的安排, 让学 生能循序渐进的掌握相关知识。

3.1 首先应阐明对于潜艇耐压圆柱壳为何在强度校核中其 稳定性往往成为影响其下潜深度因素中更为重要的因素: 在潜艇结构设计与计算中, 不但要保证壳体应力小于规定 的许用应力值, 也要保证壳体具有足够的稳定性, 由于现 代潜艇越来越多的使用 $\sigma \mathrm{s}$ 更高的强度钢以减轻耐压壳体 的重量, 因而保证船体的稳定性问题也就越加重要。

3.2 进而分析对于潜艇耐压结构而言, 其失稳的主要形式: (1)壳板局部失稳; (2)中间支骨失稳; (3)总体失稳模式。在 应用结构力学中李兹法推导得出潜艇耐压圆柱壳各种失 稳模式时其失稳临界压力理论计算公式从而让本科生对 潜艇耐压结构的失稳特性有基本的了解, 为进一步的学习 打下基础。

3.3 分析长舱段环肋圆柱壳其稳定性与普通环肋圆柱壳的 不同之处, 即: 其总体稳定性劣与局部稳定性。在潜艇设 计中, 为了提高环肋圆柱壳的临界压力, 通常主要采用加 大肋骨刚度、缩短舱室长度和加设特大肋骨 (本质上也是 缩短总稳定性计算中舱室长度的一种手段)等方法都可以 得到很好的效果。但是, 当采用高强度材料以后, 随着圆 柱壳直径加大和钢材屈服极限的提高, 圆柱壳板的相对厚 度减小, 继续采用加大肋骨刚度和减小计算跨度并不能一 直解决总体稳定性的问题, 此时结构出现所谓的“异常特 性”, 其表现有: 当肋骨刚度成倍提高, 失稳跨度成倍减 小, 总稳定性欧拉应力 $P \mathrm{E}$ 几乎不变, 失稳时纵向半波数 $\mathrm{m} \neq 1$ 等现象 [5-7]。

3.4 本课程在前面相关内容的学习后，应重点分析潜艇耐 压壳体设计为何对出现“异常特性”长舱段圆柱壳其稳定 性采用加大壳板厚度, 缩小舱室长度, 增加肋骨尺寸等常 规加强方式是无效的, 使学生对“异常特性”有更加深刻的 认识;

受均匀外压力作用的环肋圆柱壳可以看作是同时受到轴 向压力 $P_{E}^{(1)}$ 和周向压力 $P_{E}^{(2)}$ 的共同作用, 出现“异常特 性”的本质原因就是环肋圆柱壳的轴向刚度不足。因此需 要对仅受轴向压力时的理论临界压力 $P_{E}^{(1)}$ 和仅受周向压 力时的理论临界压力 $P_{E}^{(2)}$ 分别进行研究。

在《潜艇强度》课程中, 由里兹法推导的环肋圆柱壳总体 稳定性计算公式[1]为:

本科生课程除了让学生掌握扎实的学科知识外, 还要在教 学的内容上与时俱进,教学内容的选择要体现新时代创新 需要, 要以提高本科生的教育质量为目标。要让本科生了 解现代大型潜艇的使用需求以及设计过程中的新思路, 了

$$
T_{1} m^{2} \alpha^{2}+T_{2}\left(n^{2}-1\right)=\frac{D}{R^{2}}\left(m^{2} \alpha^{2}+n^{2}-1\right)^{2}+\frac{E t m^{4} \alpha^{4}}{\left(m^{2} \alpha^{2}+n^{2}\right)^{2}}+\frac{E I}{R^{2} l}\left(n^{2}-1\right)^{2}
$$


$T_{1}=0.5 P^{(1)} R, T_{2}=P^{(2)} R$ 。 $P^{(1)}$ 和 $P^{(2)}$ 分别表示圆 柱壳在轴向受到的均匀外压和周向受到的均匀外压 [5]。 仅受轴压时的总体失稳压力方程:
由公式左侧可以看出, 这个总体稳定性的临界载荷 $P \mathrm{E}$ 与 $T 1$ 和 $T 2$ 有关, 而 $T 1$ 和 $T 2$ 正是引起结构发生失稳的两种 载荷, 一个代表了轴向压力, 另外一个代表了周向压力。

$$
P_{E}^{(1)}=\frac{2 E \times 10^{-6}}{m^{2} \alpha^{2}}\left[\frac{\gamma^{3}}{12\left(1-\mu^{2}\right)}\left(m^{2} \alpha^{2}+n^{2}-1\right)^{2}+\frac{\gamma m^{4} \alpha^{4} \times 10^{4}}{\left(m^{2} \alpha^{2}+n^{2}\right)^{2}}+\beta\left(n^{2}-1\right)^{2}\right]
$$

仅受周向时的总体失稳压力方程:

$$
P_{E}^{(2)}=\frac{E \times 10^{-6}}{n^{2}-1}\left[\frac{\gamma^{3}}{12\left(1-\mu^{2}\right)}\left(m^{2} \alpha^{2}+n^{2}-1\right)^{2}+\frac{\gamma m^{4} \alpha^{4} \times 10^{4}}{\left(m^{2} \alpha^{2}+n^{2}\right)^{2}}+\beta\left(n^{2}-1\right)^{2}\right]
$$

为了讨论耐压结构的异常特性, 引入两个参数:

$$
\alpha=\frac{\pi R}{L}, \quad \beta=\frac{10^{6} I}{R^{3} l}
$$

这两个参数代表了舱段长度和周向刚度对圆柱壳结构总 体稳定性的影响。受各向均匀的外压作用下, 长舱段耐压 结构总体失稳临界压力应满足: $P_{E}<P_{E}^{(1)}, P_{E}<P_{E}^{(2)}$ 。 通过分别做出方程（1），（2）和（3）的曲线可以看出, 当 $\alpha$ 小于某一临界值 $\alpha_{i}$ 时, 舱段总体稳定性 $P_{E}$ 主要受 到 $P_{E}^{(2)}$ 的制约, 随着 $\alpha$ 的增大, 当 $\alpha>\alpha_{i}$ 时, 总体稳定 性 $P_{E}$ 受到 $P_{E}^{(1)}$ 的制约, 并且 $P_{E}$ 不再增大; 当 $\beta$ 小于某 一临界值 $\beta_{i}$ 时, 舱段总体稳定性 $P_{E}$ 主要受到 $P_{E}^{(2)}$ 的制 约, 随着 $\beta$ 的增大, 当 $\beta>\beta_{i}$ 时, 总体稳定性 $P_{E}$ 受到 $P_{E}^{(1)}$ 的制约, 并且 $P_{E}$ 不再增大。

对于常规潜艇环肋圆柱壳结构而言, 在 $\alpha<\alpha_{i}, \beta<\beta_{i}$ 的结构参数范围内, 总体稳定性规律 $P_{E}$ 与 $P_{E}^{(2)}$ 特性一 致, 主要变现为 $P_{E}$ 随着壳体周向刚度的增加而增大, 纵 向失稳半波数 $m=1$, 表现为壳体的“异常特性”, 一旦结 构参数超过此范围, 则表现为壳体与仅受轴向外压作用时 一直, 称之为“异常特性”, 这就是异常特性的本质。值得 指出的是, 纵向失稳半波数 $m \neq 1$ 并不一定舱段是判断处 于“异常特性”时的衡量标志。

异常特性对耐压结构设计最主要的启示是: 为改善环肋圆 柱壳的总体稳定性而减小舱段长度 $L$ 和增大环肋尺寸是 毫无作用的。同时由这一特性可知 [6]:

(1) 当舱段处于 “异常特性”时, 其内设置的特大肋骨 对改善舱段总体稳定性几乎无有效贡献。舱段内设置特大 肋骨, 主要作用就是利用其大刚度的作用起到减小失稳跨 距或提高周向刚度以期待提高壳体稳定性, 而“异常特性” 使得减小失稳跨距等方式无用, 故通常使用的加设特大肋 骨在此情况下也是失效的, 若忽视了“异常特性”的存在, 反而在无效的方案上更不利于舱段总布置, 得不偿失。

(2) 舱段内壳板与环肋总体稳定性临界压力等于甚至 小于其孤立环肋的失稳临界压力。当失稳跨距 $L$ 无限长 时, 其孤立肋骨的失稳临界压力就是其总体失稳临界压 力。但由于舱段 $L$ 是有限长的, 通常情况下舱段理论压 力大于孤立肋骨临界压力。但处于“异常特性”, 由于减小 跨距是无效的, 故环肋圆柱壳总体失稳临界压力等于其孤 立肋骨失稳临界压力。

3.5 在前阐述的基础上探讨潜艇耐压结构出现“异常特性”
合适且有效的加强方式: 设置纵向肋骨。

\section{4. 有限元仿真计算对分析潜艇耐压结构稳定性 的应用}

有限元分析的最大特点就是标准化和规范化, 这种特点使 得大规模分析和计算成为可能,当采用了现代化的计算机 以及所编制的软件 ABAQUS、ANAYS 等作为实现平台 时, 则复杂工程问题的大规模分析就变为了现实。正因此, 在现代潜艇的设计过程中也大量使用有限元分析帮助设 计人员更好的预报结构的力学性能, 同时开展相应的优化 设计 [8]。但本书中并未涉及潜艇耐压结构有限元分析等 相关内容的教学, 在时代性上已稍显不足。同时, 通过有 限元分析能够直观的展示潜艇舱段在巨大静水压力作用 下的失稳模态, 提高学生应用先进技术解决潜艇结构设计 过程中出现问题的能力, 有益于学生从知识的感性认识逐 渐提高到对理论知识的理性认识。

在本课程中通过教授学生潜艇耐压结构基本的有限元建 模方法, 让学生掌握基本的有限元分析过程, 将有限元分 析和《潜艇结构设计计算方法》中相关规范内容结合, 体 会在真实的设计过程中如何有效且合理的设计潜艇耐压 结构参数, 使学生能真正的应用所学知识解决问题, 体会 学习的获得感, 从而激发学生学习兴趣。

\section{5. 工程案例分析}

潜艇强度中关于耐压结构的稳定性分析根本目的是工程 设计和工程应用, 因此结合有限元和规范的稳定性分析方 法, 使学生掌握该分析方法是船舶与海洋工程专业从事潜 艇结构研究设计方向的本科生的基本要求之一。这一目标 可以通过布置大作业的形式, 要求学生根据规范内容对潜 艇耐压结构进行稳定性分析与校核, 同时通过有限元分析 进行验证的方式来实现。通过实艇耐压结构的稳定性的分 析, 学生可以更加明白潜艇耐压结构设计中稳定性校核的 重要性, 并进一步深化理解潜艇耐压结构稳定性校核的一 般流程。通过现有理论与有限元仿真计算, 让学生能将理 论知识与工程实践相结合, 真正的实现学以致用, 使掌握 的理论知识更加牢固, 既锻炼了工程实践的动手能力、提 升分析问题与解决问题的能力, 也强化了学习的目的性。 


\section{6. 结论}

我们依据自己多年的一线教学经验和科研工作经历, 对 《潜艇强度》本科生知识体系进行了初步分析与研究了, 构建了船舶与海洋工程专业《潜艇强度》这一本科生课程 的知识体系。但潜艇强度, 尤其是潜艇耐压结构稳定性分 析也是一个不断与时俱进, 随时代发展的研究热点, 必将 有一些创新成果被人们所不断探究认识。因此, 船舶与海 洋工程专业本科生课程《潜艇强度》的知识体系建构也是 一个不断完善与发展的过程, 是一个开放的体系, 有待不 断更新。

\section{致谢}

本文为黑龙江教育科学规划课题《水下耐压壳体结构力学 方向课程体系改革研究》(编号: GBC1317173) 和《船舶 与海洋工程结构力学方向课程体系改革研究》 (编号:

GJC1316080)的阶段性成果之一。

\section{参考文献}

[1] 石德新, 王晓天. 潜艇强度[M]. 哈尔滨工程大学出版 社.1997.

[2] 王晓天, 许辑平. 大型潜艇总稳定性计算中异常特性 的研究 [J].中国造船,1993,(3):36-46.

[3] 王晓天, 高艳, 许辑平. 大型潜艇总稳定性计算中出 现异常特性的研究 [J].中国造船, 1990(3):36-46

[4] 中华人民共和国国家军用标准, 潜艇结构设计计算方 法 (GJB/Z 21A-2011) [Z]. 国防科学技术工业委员会. 2011. [5] 高灵芝. 大型深潜潜艇耐压船体结构设计研究 [D]. 哈尔滨工程大学硕士学位论文. 2003.

[6] 阮俊杰. 长舱段耐压圆柱壳结构稳定性研究[D]. 哈 尔滨工程大学硕士学位论文. 2019.

[7] 阮俊杰, 许维军, 陈天一. 长舱段环肋圆柱壳稳定性 加强方式研究 [J]. 海洋工程装备与技术, 2018,10(5):244-247.

[8] 宋世伟, 张二, 吴梵. 基于 ABAQUS 的环肋圆柱壳长 舱段稳定性分析与结构优化 [J]. 船海工程, 2011,12(40):79-82. 